\section{Sensational mechanics}

Many senses that are based on mechanosensation, such as proprioception and touch, are known to be mediated by force-sensitive ion channels, but the identity of these channels has proved elusive. Now, two papers in Neuron report findings that directly implicate transient receptor potential cation channel N1 (TRPN1; also known as NompC and TRP4) in mechanosensation by fruitflies and nematode worms.

Cheng et al. showed the importance of TRPN1 for proprioception in Drosophila melanogaster. Using a green fluorescent protein construct, the authors showed expression of the channel in subsets of peripheral sensory neurons that had previously been implicated in coordinating locomotion.

TRPN1-null larvae crawled more slowly than wild-type larvae, suggesting impaired proprioception given the loss of TRPN1 in sensory neurons, and TRPN1-null adult flies exhibited uncoordinated leg and wing movements, and were unable to walk or fly. These defects were rescued by expression of TRPN1 under the control of sensory-neuronspecific promoters.

To directly observe the in vivo effects of TRPN1, the authors expressed a $\mathrm{Ca}^{2+}$ sensor in a larval preparation that maintains peristaltic muscle contractions. In fruitflies, sensory neurons in each body segment project to the corresponding segment of the ventral nerve cord (VNC) in the CNS. Muscle contractions propagating from posterior to anterior segments generated $\mathrm{Ca}^{2+}$ signals in sensory neurons that were relayed to the VNC, representing a sensory response to muscle contractions. In TRPN1-null preparations, this segmental propagation of the $\mathrm{Ca}^{2+}$ signal in the VNC was abolished. Interestingly, this function of TRPN1 in proprioception seems to be conserved, as previous work in Caenorhabditis elegans also revealed a crucial role for the receptor in this process.

Kang et al. focused on the C. elegans ciliated mechanosensory neuron known as CEP, which is located in the nose tip of the worm and is involved in the slowing of movement in response to touching food particles. Removing the cuticle on the worm head to expose the CEP cell body enabled whole-cell patch clamp recordings during mechanical stimulation of the nose tip. The rapid activation and latency times of the recorded mechanoreceptor current suggested that it is mediated by a mechanically gated channel. This current and the movement-slowing response was absent in mutant worms lacking TRPN1 and was rescued by re-expression of the channel. Crucially, to show that TRPN1 is directly responsible for the mechanoreceptor current, the authors mutated key residues in the putative pore-forming domain of the channel protein and showed that this changed the cation-selectivity of the current.
Together, these findings provide compelling evidence that TRPN1 is one of the elusive force-gated sensory channels. Although this channel is not expressed in mammals, several other TRP family members are, and these studies represent an important advance in this challenging field.

Katie Kingwell

ORIGINAL RESEARCH PAPERS Cheng, L.E.

et al. The role of the TRP channel NompC in

Drosophila larval and adult locomotion. Neuron 67, 373-380 (2010) | Kang, L. et al. C. elegans TRP family protein TRP- 4 is a pore-forming subunit of a native mechanotransduction channel. Neuron 67, 381-391 (2010)

FURTHER READING Li, W. et al. A C. elegans stretch receptor neuron revealed by a mechanosensitive TRP channel homologue. Nature 440, 684-687 (2006)

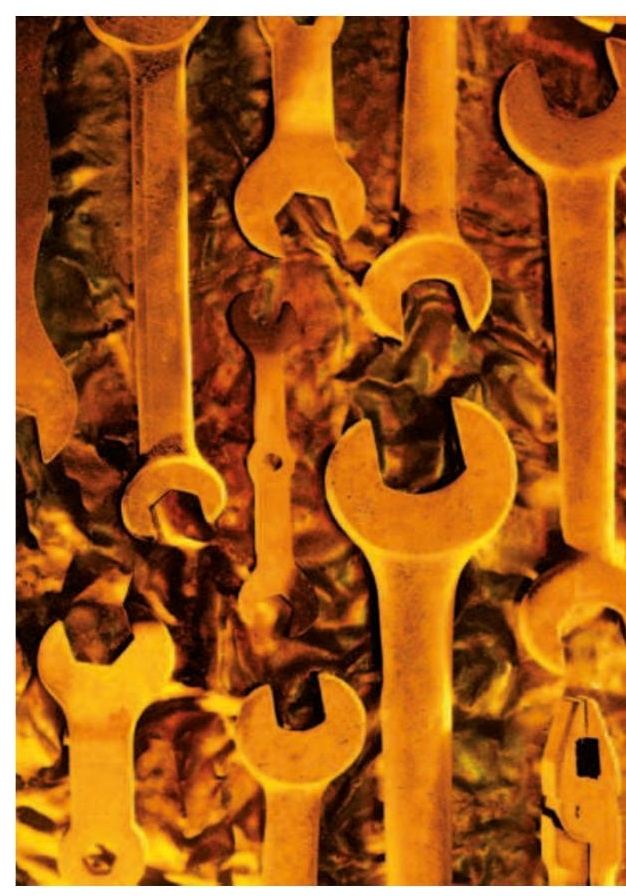

